\title{
TeV cosmic-ray proton and helium spectra in the myriad model
}

\author{
G. Bernard ${ }^{1}$, T. Delahaye ${ }^{2}$, Y.-Y. Keum ${ }^{3,4}$, W. Liu ${ }^{5}$, P. Salati ${ }^{1}$, and R. Taillet ${ }^{1}$ \\ ${ }^{1}$ LAPTh, Université de Savoie, CNRS, BP 110, 74941 Annecy-le-Vieux, France \\ e-mail: taillet@lapp.in2p3.fr \\ 2 Instituto de Física Teórica UAM/CSIC, Universidad Autónoma de Madrid, Cantoblanco, 28049 Madrid, Spain \\ 3 BK-21 Research Group, Department of Physics, Seoul National University, 157-742 Seoul, Republic of Korea \\ 4 Institute of the Early Universe, Ewha Womans University, 120-750 Seoul, Republic of Korea \\ 5 Cosmic Dark-Energy and Dark-Matter Research Group, National Astronomical Observatories, Chinese Academy of Science, \\ 100012 Beijing, PR China
}

Received 31 January 2013 / Accepted 3 May 2013

\section{ABSTRACT}

\begin{abstract}
Context. Recent measurements of cosmic ray proton and helium spectra show a hardening above a few hundred GeV. This excess is hard to understand in the framework of the conventional models of Galactic cosmic ray production and propagation.

Aims. We propose here to explain this anomaly by the presence of local sources (myriad model).

Methods. Cosmic ray propagation is described as a diffusion process taking place inside a two-zone magnetic halo. We calculate the proton and helium fluxes at the Earth between $50 \mathrm{GeV}$ and $100 \mathrm{TeV}$. As an improvement over a similar analysis, we consistently derive these fluxes by taking both local and remote sources for which a unique injection rate is assumed into account.

Results. We find cosmic ray propagation parameters compatible with $\mathrm{B} / \mathrm{C}$ measurements for which the proton and helium spectra agree remarkably with the PAMELA and CREAM measurements over four decades in energy.
\end{abstract}

Key words. catalogs - cosmic rays - pulsars: general

\section{Introduction}

The energy spectrum of primary cosmic rays behaves approximately as $E^{-2.7}$ in the $10 \mathrm{GeV}$ to $100 \mathrm{TeV}$ range. This is fairly well understood if one assumes that these cosmic rays are accelerated by energetic events such as supernova explosion shocks, that are distributed evenly in the disk of our Galaxy. Once injected inside the Galactic magnetic halo with a rate $q \propto \mathcal{R}^{-\alpha}$ where $\mathcal{R} \equiv p / Z e$ stands for the rigidity and $\alpha \simeq$ $2.15 \pm 0.15$, particles are subsequently scattered by the turbulent irregularities of the Galactic magnetic field. Their transport is described phenomenologically by space diffusion with a coefficient $K \propto \mathcal{R}^{\delta}$ whose energy dependence is characterized by the index $\delta$. The boron-to-carbon ratio $(\mathrm{B} / \mathrm{C})$ is a tracer of cosmic ray (CR) propagation that points towards a value of $\delta \in[0.4,0.85]$ for the diffusion index. At high energy, the flux of a given primary CR species above the Earth is given by $\Phi \propto q / K$ and decreases with energy as $1 / E^{\alpha+\delta}$. Its spectrum exhibits power-law behavior whose index is the sum of $\alpha$ and $\delta$.

However, this is challenged by the recent measurements of the absolute high-energy $\mathrm{CR}$ proton and helium spectra that have been recently reported by CREAM (Ahn et al. 2010; Yoon et al. 2011) and PAMELA (Adriani et al. 2011) experiments. Observations indicate there is an excess in the CR proton and helium fluxes above $250 \mathrm{GeV} /$ nuc. The single power-law hypothesis is rejected at $95 \%$ C.L. The hardening of the proton spectrum occurs at $232_{-30}^{+35} \mathrm{GeV}$ with a change of the spectral index from $2.85 \pm 0.015 \pm 0.004$ to $2.67 \pm 0.03 \pm 0.05$. For the helium data, the spectral index varies from $2.766 \pm$ $0.01 \pm 0.027$ to $2.477 \pm 0.06 \pm 0.03$ with the hardening setting in at $243_{-31}^{+27} \mathrm{GeV} / \mathrm{nuc}$.
Explanations of this anomaly have been tentatively given ever since its discovery. They mainly imply a modification of the energy behavior of either the injection spectrum $q(E)$ or the diffusion coefficient $K(E)$. To begin with, a break in $\alpha$ could arise from a modification of the conventional diffusive shock acceleration (DSA) scheme, as suggested by Malkov et al. (2012) and Ohira \& Ioka (2011). In the same vein, the possibility of different classes of CR sources has been proposed some time ago by Stanev et al. (1993) and Zatsepin \& Sokolskaya (2006); for instance, cosmic rays accelerated in the magnetized winds of exploding Wolf-Rayet and red supergiant stars could have a double spectrum, with a hard component produced in the polar cap regions of these objects. According to Biermann et al. (2010), this hard component would take over the smooth one above a few hundred $\mathrm{GeV}$, hence the observed break in the proton and helium spectra. Not so different is the proposition of Yuan et al. (2011) where a spread in the injection index $\alpha$ is introduced.

Another direction implies a modification of the diffusion coefficient $K$. As proposed by Ave et al. (2009), the proton and helium anomaly could be due to a welcome, but unexpected, decrease in the spectral index $\delta$ at high energy. Recently Blasi et al. (2012) have offered some theoretical motivations to such changes in diffusion. A local variation in $K$ could also have a similar effect, as suggested by Tomassetti (2012). Finally, inspired by Hörandel et al. (2007), Blasi \& Amato (2011) invokes an unusually strong spallation of the $\mathrm{CR}$ species on the Galactic gas. This possibility has been recently criticized in a detailed analysis carried out by Vladimirov et al. (2012) of some of the above-mentioned solutions to the CR proton and helium anomaly.

This paper computes the proton and helium spectra within the usual framework of diffusive propagation, and we use the 
Table 1. Characteristics of nearby supernova remnants.

\begin{tabular}{|c|c|c|c|c|c|c|}
\hline $\begin{array}{l}\text { SNR } \\
\text { G+long+lat }\end{array}$ & Other name & $\begin{array}{l}\text { Distance } \\
{[\mathrm{kpc}]}\end{array}$ & $\begin{array}{c}\text { Radio index } \\
\sigma_{r}\end{array}$ & $\begin{array}{c}\text { Brightness } \\
\text { [Jy] }\end{array}$ & $\begin{array}{l}\text { Age } \\
{[\mathrm{kyr}]}\end{array}$ & Pulsar \\
\hline $18.95-1.1$ & & 2. $\pm \mathbf{0 . 1}$ & 0.28 & 40 & $11.75 \pm 0.85$ & $?$ \\
\hline $65.3+5.7$ & & $0.9 \pm 0.1$ & $0.58 \pm 0.07$ & 52 & $26 \pm 1$ & $\emptyset$ \\
\hline $65.7+1.2$ & DA 495 & $1.0 \pm 0.4$ & $0.45 \pm 0.1$ & 5 & $16.75 \pm 3.25$ & unknown \\
\hline $69.0+2.7$ & СТВ 80 & $2.0 \pm \mathbf{0 . 1}$ & $0.20 \pm 0.10$ & $60 \pm 10$ & $20 \pm 1$ & $\mathrm{~J} 1952+3252$ \\
\hline $74.0-8.5$ & Cygnus Loop & $0.54_{-0.08}^{+0.10}$ & $0.4 \pm 0.06$ & $175 \pm 30$ & $10 \pm 1$ & $\emptyset$ \\
\hline $78.2+2.1$ & $\gamma$ Cygni & $1.5 \pm \mathbf{0 . 1}$ & $0.75 \pm 0.03$ & $275 \pm 25$ & $7 \pm \mathbf{1}$ & $\emptyset$ \\
\hline $82.2+5.3$ & W63 & $2.3 \pm 1.0$ & $0.36 \pm 0.08$ & $105 \pm 10$ & $20.1 \pm 6.6$ & $\emptyset$ \\
\hline $89.0+4.7$ & HB 21 & $1.7 \pm 0.5$ & $0.27 \pm 0.07$ & $200 \pm 15$ & $5.60 \pm 0.28$ & $\emptyset$ \\
\hline $93.7-0.2$ & CTB 104A / DA 551 & $1.5 \pm 0.2$ & $0.52 \pm 0.12$ & $42 \pm 7$ & $50 \pm 20$ & $\emptyset$ \\
\hline $114.3+0.3$ & & 0.7 & $0.49 \pm 0.25$ & $6.4 \pm 1.4$ & $7.7 \pm \mathbf{0 . 1}$ & $\emptyset$ \\
\hline $116.5+1.1$ & & 1.6 & $0.16 \pm 0.11$ & $10.9 \pm 1.2$ & $20 \pm 5$ & B $2334+61 ?$ \\
\hline $116.9+0.2$ & СТВ 1 & 1.6 & $0.33 \pm 0.13$ & $6.4 \pm 1.4$ & $20 \pm 5$ & B2334 + 61? \\
\hline $119.5+10.2$ & CTA 1 & $1.4 \pm 0.3$ & $0.57 \pm 0.06$ & $42.5 \pm 2.5$ & $10 \pm 5$ & $\mathrm{~J} 0010+7309$ \\
\hline $127.1+0.5$ & R5 & $1 . \pm 0.1$ & $0.43 \pm 0.1$ & $12 \pm 1$ & $25 \pm 5$ & $\emptyset$ \\
\hline $156.2+5.7$ & & $0.8 \pm 0.5$ & $2.0_{-0.7}^{+1.1}$ & $4.2 \pm 0.1$ & $10 \pm 1$ & $\mathrm{~B} 0450+55 ?$ \\
\hline $160.9+2.6$ & HB 9 & $0.8 \pm 0.4$ & $0.48 \pm 0.03$ & $\sim 75$ & $5.5 \pm 1.5$ & B0458 + 46 \\
\hline $180.0-1.7$ & S147 & $1.2 \pm 0.4$ & 0.75 & $74 \pm 12$ & $600 \pm \mathbf{1 0}$ & $\mathrm{J} 0538+2817$ \\
\hline $184.6-5.8$ & Crab / 3C144 / SN1054 & $2.0 \pm 0.5$ & 0.3 & 1040 & $7.5 \star$ & B0521 + 31 \\
\hline $189.1+3.0$ & IC 443 & $1.5 \pm \mathbf{0 . 1}$ & $0.36 \pm 0.04$ & $160 \pm 5$ & 30 or 4 & $\emptyset$ \\
\hline $203.0+12.0$ & Monogem ring & $0.288_{-0.027}^{+0.033}$ & & & $86 \pm \mathbf{1}$ & B $0656+14$ \\
\hline $205.5+0.5$ & Monoceros nebula & $1.63 \pm 0.25$ & $0.66 \pm 0.2$ & $156.1 \pm 19.9$ & $29 \pm 1$ & $\emptyset$ \\
\hline $263.9-3.3$ & Vela(XYZ) & $0.295 \pm 0.075$ & variable & $2000 \pm 700$ & $11.2 \pm \mathbf{0 . 1}$ & B0833-45 \\
\hline $266.2-1.2$ & Vela Jr / SN1300 & $0.75 \pm \mathbf{0 . 0 1}$ & & & $3.5 \pm 0.8 \star ?$ & J0855-4644? \\
\hline $276.5+19.0$ & Antlia & $0.2 \pm 0.14$ & & & $\geq 1000$ & B0950+ 08 \\
\hline $315.1+2.7$ & & $1.7 \pm 0.8$ & 0.7 & & $50 \pm 10$ & $\mathrm{~J} 1423-56$ \\
\hline $330.0+15.0$ & Lupus Loop & $0.8 \pm 0.1$ & & & $50 \pm 10$ & B1507-44? \\
\hline $347.3-0.5$ & SN393 & $1 . \pm 0.3$ & & & $4.9 \star$ & $\emptyset$ \\
\hline $73.90+0.90$ & & $1.5 \pm 0.5$ & & & $11.5 \pm 0.50$ & $\emptyset$ \\
\hline $107.50-1.50$ & & 1.1 & & & 4.5 & $\emptyset$ \\
\hline $272.20-3.20$ & & $3.4 \pm 1.6$ & & & $8.5 \pm 0.1$ & $\emptyset$ \\
\hline
\end{tabular}

Notes. Spectral index and brightness are inferred from measurements made at $1 \mathrm{GHz}$. Uncertainties in bold are not taken from bibliographic references, but just correspond to a rough uncertainty on the last relevant digit, so they can be underestimated. Age is flagged with a $\star$ for a historical remnant; in this case, the age uncertainty is set from the distance uncertainty. Except for the historical supernovæ, the ages are the observed ages, which differ from the actual ages by $d / c$.

propagation parameters consistent with $\mathrm{B} / \mathrm{C}$ spectra, while taking the known local sources of cosmic rays into account. We build a catalog of nearby and recent sources (see Table 1), because the known supernova remnants (SNR) and pulsars to which they are associated can be found in astronomical catalogs such as the Green catalog (Green 2009) and the ATNF pulsar database (Manchester et al. 2005). This catalog is almost the same as the one used in Bernard et al. (2012) to illustrate the importance of considering local sources, for one particular set of diffusion parameters. In this paper, we go a step further to explore all the propagation parameters consistent with the observed $\mathrm{B} / \mathrm{C}$ at low energy.

Our goal is to investigate whether one can set new constraints on these parameters when using the proton and helium spectra observed by PAMELA and CREAM. We show that the proton and helium spectral hardening above $250 \mathrm{GeV} /$ nuc can indeed be attributed to the known local sources of cosmic rays. In our approach, there is no need to modify the conventional CR propagation model. In particular, the variations with CR energy in the injection rate $q$ of individual sources and of the space diffusion coefficient $K$ are power laws that are characterized by the spectral indices $\alpha$ and $\delta$ respectively. This idea has already been suggested recently by Erlykin \& Wolfendale (2011), who explain the hardening with very few sources (mainly Monogem Ring), and by Thoudam \& Hörandel (2012) who consider a catalog of ten nearby sources. The principal weakness of these analyses is the lack of a consistent treatment of the CR spectra in the entire energy range extending from tens of $\mathrm{GeV}$ up to a few $\mathrm{PeV}$. This is particularly clear in Thoudam \& Hörandel (2012) where the proton and helium anomaly is derived from a handful of local sources, whereas the low-energy spectra of these species are not calculated but merely fitted in order to get a value for $\alpha$ once $\delta$ has been chosen. It should be noted that the magnitude of the CR proton (helium) flux is related over the entire energy range to the injection rate $q$ of individual sources. The low-energy (power-law regime) and high-energy (spectral hardening) parts of the CR spectra are connected to each other. A consistent treatment of the problem requires that the proton and helium fluxes are calculated over the entire energy range. A crucial problem is also to understand why just a few local sources could explain the spectral hardening at high energies, whereas the bulk of the Galactic sources is required in order to account for the power-law behavior of the fluxes below $250 \mathrm{GeV} /$ nuc. This aspect, which is not addressed in the above-mentioned analyses, bears upon the more general question of the discrete nature of the sources. In the conventional model of CR propagation, these are treated as a jelly spreading over the Galactic disk and continuously accelerating cosmic rays. The question arises then as to why and in which conditions that scheme breaks down at high energies where local and discrete objects come into play.

The results presented here are based on a detailed investigation of that question. Bernard et al. (2012) have recently shown 
how to reconcile the presence of discrete sources with the conventional description of $\mathrm{CR}$ production and propagation, in a more theoretical analysis. We briefly recall the salient features of their analysis, before extending it to the general study, leaning on the use of the catalog shown in Table 1.

\section{The proton and helium anomaly in light of local sources}

\subsection{Cosmic ray propagation with discrete sources}

Once accelerated by the sources that lie within the Galactic disk, CR nuclei diffuse, due to scattering on the irregularities of the Galactic magnetic field. The diffusion coefficient $K=$ $K_{0} \beta \mathcal{R}^{\delta}$ accounts for that process, where $K_{0}$ is a normalization constant and $\beta$ denotes the particle velocity. The magnetic halo $(\mathrm{MH})$ inside which cosmic rays propagate before escaping into intergalactic space is assumed to be a flat cylindrical domain that matches the circular structure of the Milky Way. The Galactic disk is sandwiched between two confinement layers whose thickness $L$ is unknown, and it turns out to be crucial in our investigation. Stellar winds combine to generate a Galactic convection that wipes cosmic rays away from the disk, with velocity $V_{\mathrm{c}}(z)=V_{\mathrm{c}} \operatorname{sign}(z)$. The CR nuclei also undergo collisions with the interstellar medium (ISM) at a rate

$\Gamma_{\mathrm{sp}}=\beta\left(\sigma_{\mathrm{pH}} n_{\mathrm{H}}+\sigma_{\mathrm{pHe}} n_{\mathrm{He}}\right)$,

where the densities $n_{\mathrm{H}}$ and $n_{\mathrm{He}}$ have been respectively averaged to 0.9 and $0.1 \mathrm{~cm}^{-3}$. The total proton-proton cross section $\sigma_{\mathrm{pH}}$ has been parameterized according to Nakamura et al. (2010), while $\sigma_{\mathrm{pHe}}$ is related to $\sigma_{\mathrm{pH}}$ by the Norbury \& Townsend (2007) scaling factor $4^{2.2 / 3}$. Similar scaling factors have been used to derive the $\mathrm{CR}$ helium nuclei collision cross sections from the proton case. Above a few $\mathrm{GeV}$, diffusive re-acceleration and energy losses may be disregarded and the master equation for the space and energy number density $\psi \equiv \mathrm{d} n / \mathrm{d} T$ of a given $\mathrm{CR}$ species simplifies into the diffusion equation

$\frac{\partial \psi}{\partial t}+\partial_{z}\left(V_{\mathrm{c}} \psi\right)-K(E) \Delta \psi+\Gamma_{\mathrm{sp}} \psi=q_{\mathrm{acc}}$.

The CR transport parameters $K_{0}, \delta, L$ and $V_{\mathrm{c}}$ can be constrained from the B/C - see for instance Maurin et al. (2001), Putze et al. (2010) and Maurin et al. (2010). Our solution to the master Eq. (2) is based on the existence of a Green function $\mathcal{G}$ and is well adapted to the presence of discrete and extended CR sources. As an illustration, the CR proton density at the Earth may be expressed as the convolution over space and time of the Green function $\mathcal{G}_{\mathrm{p}}$ with $q_{\text {acc }}$

$\psi(\boldsymbol{x}, t)=\int_{-\infty}^{t} \mathrm{~d} t_{\mathrm{S}} \int_{\mathrm{MH}} \mathrm{d}^{3} \boldsymbol{x}_{\mathrm{S}} \mathcal{G}_{\mathrm{p}}\left(\boldsymbol{x}, t \leftarrow \boldsymbol{x}_{\mathrm{S}}, t_{\mathrm{S}}\right) q_{\mathrm{acc}}\left(\boldsymbol{x}_{\mathrm{S}}, t_{\mathrm{S}}\right)$,

where $q_{\mathrm{acc}}\left(\boldsymbol{x}_{\mathrm{S}}, t_{\mathrm{S}}\right)$ is the CR proton injection rate at the source located at $\boldsymbol{x}_{\mathrm{S}}$ and at time $t_{\mathrm{S}}$. The propagator $\mathcal{G}_{\mathrm{p}}$ translates the probability for a CR proton injected at position $\boldsymbol{x}_{\mathrm{S}} \equiv\left(x_{\mathrm{S}}, y_{\mathrm{S}}, z_{\mathrm{S}}\right)$ and time $t_{\mathrm{S}}$ to travel through the Galactic magnetic fields until it reaches, at time $t$, an observer located at $\boldsymbol{x} \equiv(x, y, z)$.

In the conventional approach, the CR source term $q_{\text {acc }}$ is a continuous function of space and time. Steady state is moreover assumed. This is an oversimplification insofar as CR sources are actually discrete, with an average supernova explosion rate $v$ of 0.8 to 3 events per century (Diehl et al. 2006). In the stochastic treatment developed by Bernard et al. (2012), sources are modeled as point-like objects, and the production rate of CR nuclei through acceleration is given by

$q_{\mathrm{acc}}\left(\boldsymbol{x}_{\mathrm{S}}, t_{\mathrm{S}}\right)=\sum_{i \in \mathcal{P}} q_{i} \delta^{3}\left(\boldsymbol{x}_{\mathrm{S}}-\boldsymbol{x}_{i}\right) \delta\left(t_{\mathrm{S}}-t_{i}\right)$,

where each source $i$ that belongs to the population $\mathcal{P}$ contributes a factor $q_{i}$ at position $\boldsymbol{x}_{i}$ and time $t_{i}$. The total flux $\Phi \equiv(1 / 4 \pi) \beta \psi$ at the Earth depends on the precise locations and ages of all the sources and varies from one particular population $\mathcal{P}$ to another. Because we do not know the actual distribution of the Galactic sources that have generated the observed CR flux, we must rely on a statistical analysis and consider the position and age of each source as random variables. The CR flux $\Phi(E)$ at a given energy $E$ behaves as a stochastic variable whose probability distribution function $p(\Phi)$ has been studied in Bernard et al. (2012). The conventional CR model is recovered by taking the statistical average of the flux over the ensemble of all possible populations $\mathcal{P}$. This average flux $\bar{\Phi}$ turns out to be the solution of Eq. (2) with a continuous source term $q_{\text {acc }}$. More exciting is the spread of the flux $\Phi$ around its average value $\bar{\Phi}$. Using a Monte Carlo approach, Bernard et al. (2012) have shown that if the magnetic halo is thin, the statistical fluctuations of the flux may be significant. The residence time $\tau_{\text {dif }} \sim L^{2} / K$ of CR nuclei within the magnetic halo decreases with its thickness $L$. The number $N$ of sources that contribute to the signal above the Earth scales as $v \tau_{\text {dif }}$. The smaller $L$, the smaller $N$ and the larger the flux variance. Should the magnetic halo be thin enough, we expect fluctuations in the flux, especially at high energies where $K$ becomes large. In this case, the hardening of the proton and helium spectra appears to be a mere fluctuation of the CR flux, and its probability to occur is not vanishingly small.

According to this line of reasoning, the proton and helium anomaly results from the particular configuration of the actual CR sources. These objects are incidentally known in the nearby region for which catalogs of SNR and pulsars are available. The domain extending $2 \mathrm{kpc}$ around the Earth and encompassing objects that have exploded less than 30000 years ago is defined as the local region. The catalogs are no longer complete outside these limits and fail to be reliable. In the conventional CR model, the local sources would yield an average contribution $\bar{\Phi}_{\text {loc }}$ whereas the actual objects yield a much larger flux $\Phi_{\text {cat }}$. Denoting the flux from the other sources by $\Phi_{\text {ext }}$, we infer a total signal above the Earth

$\Phi=\Phi_{\text {cat }}+\Phi_{\text {ext }}$,

to be compared to the prediction of the conventional steady-state model

$\bar{\Phi}=\bar{\Phi}_{\mathrm{loc}}+\bar{\Phi}_{\mathrm{ext}}$.

The flux produced by the external sources has a very small variance as shown in Bernard et al. (2012). We may then identify $\Phi_{\text {ext }}$ with its statistical average $\bar{\Phi}_{\text {ext }}$.

\subsection{Scan of the parameter space}

The CR propagation parameters giving a good agreement with the secondary-to-primary $\mathrm{B} / \mathrm{C}$ measurements have been determined by Maurin et al. (2001) using the same propagation model as the one described above. For each of these 1600 different sets of parameters, CR propagation is specified by $K_{0}, \delta, V_{\mathrm{c}}$ and $L$. 
Table 2. Sets of CR injection and propagation parameters discussed in the text.

\begin{tabular}{lcccccc}
\hline \hline Model & $K_{0}\left[\mathrm{kpc}^{2} / \mathrm{yr}\right]$ & $\delta$ & $L[\mathrm{kpc}]$ & $V_{\mathrm{c}}[\mathrm{kpc} / \mathrm{yr}]$ & $q_{\mathrm{p}}^{0}\left[\mathrm{GeV}^{-1}\right]$ & $q_{\mathrm{He}}^{0}\left[(\mathrm{GeV} / \mathrm{n})^{-1}\right]$ \\
\hline A & $2.4 \times 10^{-9}$ & 0.85 & 1.5 & $1.38 \times 10^{-8}$ & $1.17 \times 10^{52}$ & $3.22 \times 10^{51}$ \\
B & $2.4 \times 10^{-9}$ & 0.85 & 1.5 & $1.38 \times 10^{-8}$ & $0.53 \times 10^{52}$ & $1.06 \times 10^{51}$ \\
MED & $1.12 \times 10^{-9}$ & 0.7 & 4 & $1.23 \times 10^{-8}$ & $15.8 \times 10^{51}$ & $3.14 \times 10^{51}$ \\
\hline model & $\alpha_{\mathrm{p}}+\delta$ & $\alpha_{\mathrm{He}}+\delta$ & $v\left[\right.$ century $\left.^{-1}\right]$ & $\mathrm{H}$ injection & He injection & $\chi^{2} /$ d.o.f. \\
\hline A & 2.9 & 2.8 & 0.8 & 0.19 & 0.05 & 0.61 \\
B & 2.85 & 2.7 & 1.4 & 0.12 & 0.07 & 1.09 \\
MED & 2.85 & 2.7 & 0.8 & 0.148 & 0.07 & 1.3 \\
\hline
\end{tabular}

Notes. The diffusion coefficient normalization $K_{0}$ and spectral index $\delta$, halo half height $L$, and convective velocity $V_{\mathrm{c}}$ describe the diffusion model. The source terms $q_{\mathrm{p}}^{0}$ and $q_{\mathrm{He}}^{0}$ and spectral source indices $\alpha_{\mathrm{p}}$ and $\alpha_{\mathrm{He}}$ describe the individual sources. The "injection" terms give the fraction of the supernova energy that is injected into cosmic ray acceleration, between $1 \mathrm{GeV}$ and $10^{6} \mathrm{GeV}$.

The injection rate of CR species $\mathrm{j}$ is assumed to generically be of the form

$q_{\mathrm{j}}(p)=q_{\mathrm{j}}^{0}\left(\frac{p}{1 \mathrm{GeV} / \mathrm{nuc}}\right)^{-\alpha_{\mathrm{j}}}$,

and to be the same for all CR sources. They are specified by the parameters $q_{\mathrm{p}}^{0}, q_{\mathrm{He}}^{0}, \alpha_{\mathrm{p}}$, and $\alpha_{\mathrm{He}}$. The final ingredient is the average supernova explosion rate $v$. We used these parameters to compute the proton and helium fluxes $\bar{\Phi}_{\text {ext }}+\Phi_{\text {cat }}$ over an energy range extending from $50 \mathrm{GeV} /$ nuc to $100 \mathrm{TeV} /$ nuc, in order to compare them with the PAMELA (Adriani et al. 2011) and CREAM (Ahn et al. 2010) data. At high energy, solar modulation has little effect on the CR flux, and it has not been taken into account in this study. The quality of the fit to the data is gauged by the proton and helium chi-squares $\chi_{\mathrm{p}}^{2}$ and $\chi_{\mathrm{He}}^{2}$. Our calculation of the local contribution $\Phi_{\text {cat }}$ to the flux is based on the Green catalog (Green 2009) and on the ATNF pulsar database (Manchester et al. 2005).

We performed a scan over the CR propagation parameters derived by Maurin et al. (2001). For a given set of these CR parameters, we adjusted the source parameters $q_{\mathrm{p}}^{0}, q_{\mathrm{He}}^{0}, \alpha_{\mathrm{p}}, \alpha_{\mathrm{He}}$, and $v$ to get the lowest value for the total chi-square $\chi_{\mathrm{p}}^{2}+\chi_{\mathrm{He}}^{2}$. We first set the injection indices $\alpha_{\mathrm{p}}$ and $\alpha_{\mathrm{He}}$, as well as the explosion rate $v$, and determined the best-fit values for the injection normalizations $q_{\mathrm{p}}^{0}$ and $q_{\mathrm{He}}^{0}$. We then varied $v$ from 0.5 to 3.5 century $^{-1}$ and the injection indices $\alpha_{\mathrm{p}}$ and $\alpha_{\mathrm{He}}$ from 1.75 to 2.35 to get the best adjustment of the source parameters to the CREAM and PAMELA data. The injection indices $\alpha_{\mathrm{p}}$ and $\alpha_{\mathrm{He}}$ are determined independently of each other. Observations point towards slightly different power laws for the proton and helium fluxes at energies below $250 \mathrm{GeV} /$ nuc.

\subsection{Grammages}

The propagation parameters described above were chosen to give the right $\mathrm{B} / \mathrm{C}$ at relatively low energy (below $\sim 10 \mathrm{GeV}$ ), in a diffusion model where the sources were assumed to be continuously distributed in the Galactic disk. This does not contradict the central hypothesis of our analysis: discrete sources. Indeed, Taillet et al. (2004) showed that the path-length distribution for discrete sources is very close to the distribution obtained for continuous sources. Moreover, at a few $\mathrm{GeV}$, the distance $r \sim \sqrt{K t}$ over which diffusion is effective for $t \lesssim 30000 \mathrm{y}$ is a few parsecs, which is much smaller than $2 \mathrm{kpc}$. This means that most sources that contribute to the $\mathrm{B} / \mathrm{C}$ at these energies are not in the region we defined as "local", or stated otherwise, the contribution of the discrete sources of our catalog to the observed $\mathrm{B} / \mathrm{C}$ is negligible at a few $\mathrm{GeV}$ and below.

\subsection{Results}

The range of spectra obtained by the scan described above is very large, with some of them much higher and others much lower than the measured spectra, for protons and helium. However, we find that some of the parameter sets compatible with $\mathrm{B} / \mathrm{C}$ give a good agreement with measurements. Model $\mathrm{A}$ yields the best $\chi^{2}$, and the resulting spectra are shown in Fig. 1, with the corresponding parameters are reproduced in Table 2. The CR parameters of model B are the same as for model A. The average supernova rate has been fixed to a value of 1.4 per century. The resulting spectra are shown in Fig. 2. Model C corresponds to the MED configuration as defined by Donato et al. (2004) and best fits the B/C. The half thickness $L$ of the magnetic halo is $4 \mathrm{kpc}$. The resulting spectra are shown in Fig. 3. The agreement with the CREAM and PAMELA proton and helium excess is still reasonable. These sets of CR injection and propagation parameters featured in this table have been shown in Maurin et al. (2001) to be compatible with the B/C, and they provide reasonable to very good fits to the PAMELA and CREAM data from $50 \mathrm{GeV} /$ nuc to $100 \mathrm{TeV} /$ nuc. The proton and helium fluxes are simultaneously adjusted with the same values of $K_{0}, \delta, L$, and $V_{\mathrm{c}}$. The injection indices $\alpha_{\mathrm{p}}$ and $\alpha_{\mathrm{He}}$ are determined independently. The average supernova explosion rate per century is denoted by $v$. The results of the fits to the proton and helium spectra are gauged by the total reduced chisquare $\chi_{\text {red }}^{2}$ (see Table 2$)$.

\section{Discussion of the results}

This excellent agreement makes us confident that the proton and helium anomaly can actually be explained by existing local sources that have been extracted from SNR and pulsar surveys. The model that we have presented here is quite simple, but refining it is beyond the scope of this paper. Some directions can nevertheless be given for improving the solution we just sketched.

\subsection{Value of $L$}

As a start, the best fits are obtained for a rather low value of the magnetic halo thickness $L$. This trend can be understood as follows. As already explained, the thinner the magnetic halo, the smaller the number $N$ of sources that contribute to the total signal and the greater the injection rate $q$ of individual sources. 


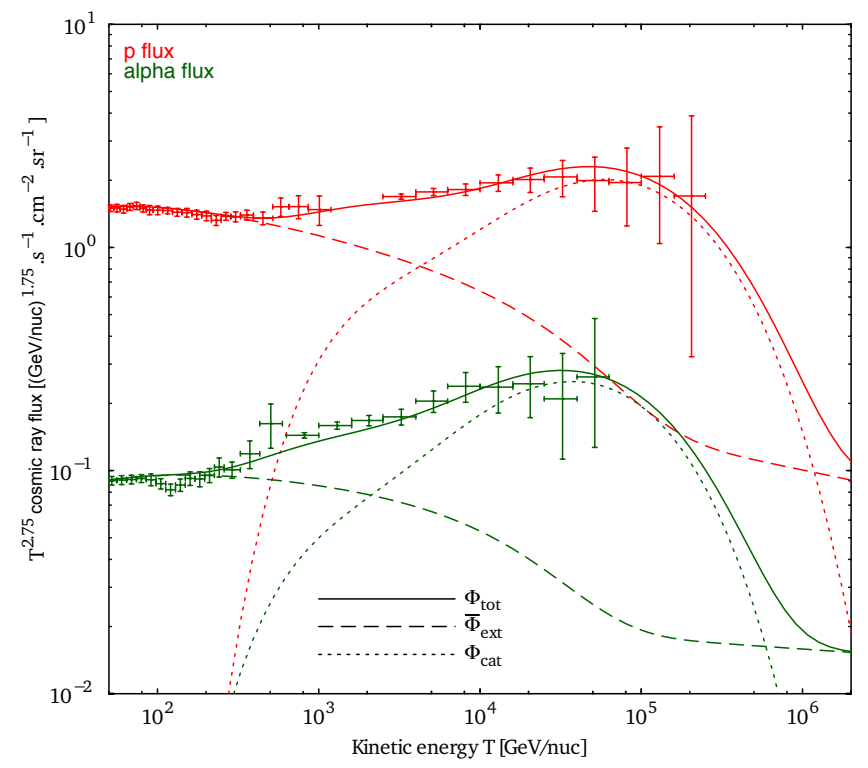

Fig. 1. Proton (upper curve) and helium (lower curve) spectra in the range extending from $50 \mathrm{GeV} /$ nuc to $100 \mathrm{TeV} /$ nuc, for the propagation parameters of model A (see Table 2), giving the best fit to the PAMELA (Adriani et al. 2011) and CREAM (Ahn et al. 2010) data: supernovae explosion rate $v=0.8$ century $^{-1}$. Solid lines show the total flux, short-dashed lines show the flux due to the sources of the catalog, and the long-dashed curve the flux due to the rest of the sources.

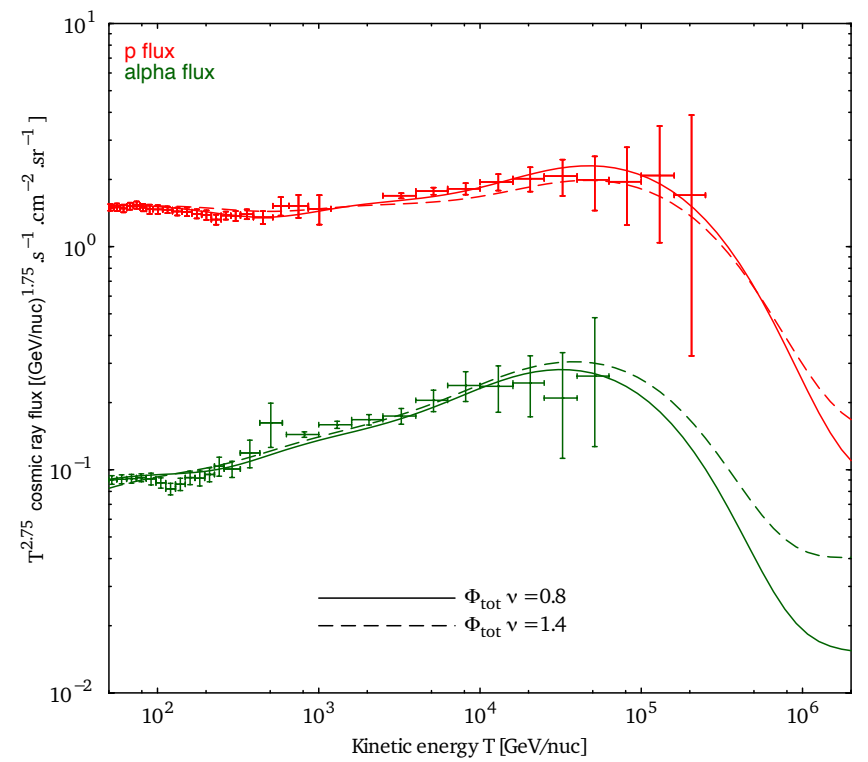

Fig. 2. Same as previous figures for models A and B (see Table 2), for two values of the supernovae explosion rate, $v=0.8$ century $^{-1}$ and $v=$ 1.4 century $^{-1}$.

The contributions $\Phi_{\text {cat }}$ and $\bar{\Phi}_{\text {loc }}$ from the local region are no longer swamped in the total flux when $L$ is low. This may be a problem since recent studies (Strong et al. 2010; Bringmann et al. 2012) of the gamma-ray and synchrotron diffuse emissions seem to favor rather high values of $L$. A possible improvement of our model would be to distribute the CR sources within spiral arms and to take into account the rotation of the Galaxy. The sources that we have considered here to derive the contribution $\bar{\Phi}_{\text {ext }}$ to the flux are equally spread along the azimuthal direction. It would be interesting to investigate whether $\bar{\Phi}_{\text {ext }}$ decreases in a more realistic setup.

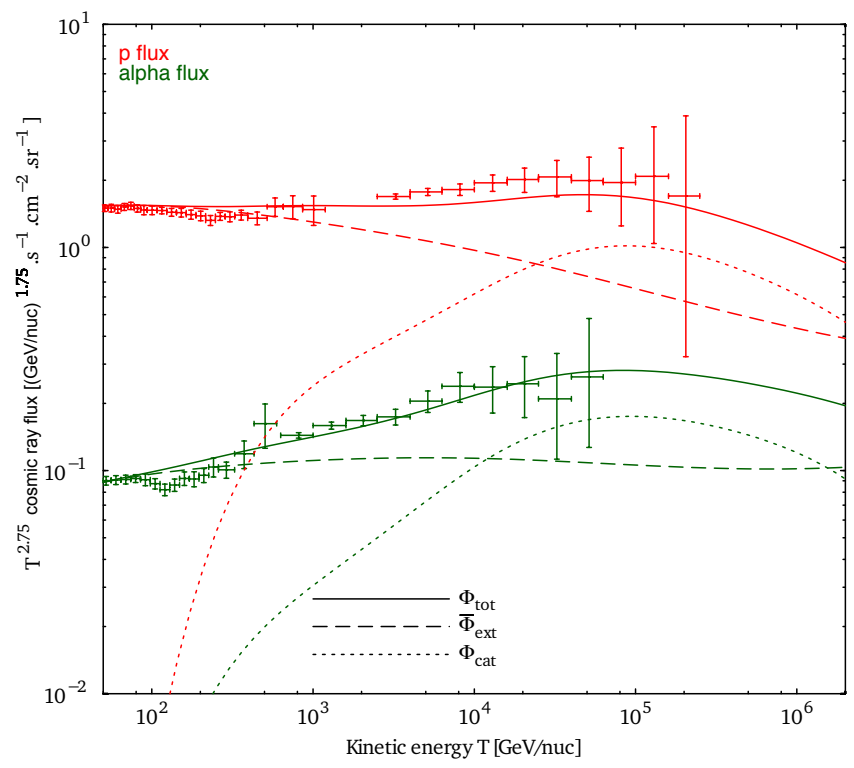

Fig. 3. Same as before, for the MED propagation parameters (see Table 2).

\subsection{Properties of supernovae}

To get a significant injection rate $q$, we are naturally driven towards a low supernova explosion rate $v$. The values found for models A and B are close to 1 explosion per century, at the lower edge of the plausible range, although not exclusively (Diehl et al. 2006).

The local sources that we have extracted from the catalogs correspond to a higher rate $v$ of 3.3 events per century. Because the Sun lies near two Galactic arms, the average explosion rate in our neighborhood could reasonably be higher than the mean rate of the Galaxy. This is actually supported by our catalog. As shown in Fig. 7 of Bernard et al. (2012), the number of known sources in our vicinity is compatible with a value of $v$ greater than three explosions per century for the past $3 \times 10^{4}$ years (depending on the radial distribution of the CR sources along the Galactic disk). Taking the Galactic spiral arms and their rotation into account could lead to a higher value of the average explosion rate and alleviate the apparent discrepancy between the average and local values of $v$.

Finally, we modeled the supernova explosions as point-like events. Cosmic rays are believed to be accelerated in the shocks that follow these explosions and which propagate in the ISM during $10^{5}$ years. The injection sites are more spherical shells than points. Depending on the CR energy, Thoudam \& Hörandel (2012) quote escape times between 500 and $10^{5}$ years after the stellar explosion. The injection takes place from a remnant whose radius varies from 5 to $100 \mathrm{pc}$. Taking the actual structure of CR accelerators into account could substantially modify the contribution $\Phi_{\text {cat }}$ to the total signal, allowing greater values of $L$ to provide acceptable fits to the PAMELA and CREAM data. The simplistic solution to the proton and helium anomaly that we have sketched in this paper is definitely promising in spite of the problems we mentioned and it should motivate further investigations.

\section{Conclusions}

Taking the discreteness of cosmic ray sources in the solar neighborhood into account, we found that the proton and helium spectra computed in a diffusion model agree with the PAMELA 
and CREAM measurements over four decades in energy, for some cosmic ray propagation parameters that are also compatible with $\mathrm{B} / \mathrm{C}$ measurements. Even if the excess at high energy happened not to be confirmed by further measurements and analysis, the proton and helium spectra could be used to put severe constraints on the parameters describing the diffusion of cosmics rays in our Galaxy. We expect that the study of the anisotropy induced by the discreteness of the sources will also provide very valuable information on the source distribution, as well as on the propagation parameters.

Acknowledgements. This work was supported by the Spanish MICINNs Consolider-Ingenio 2010 Program under grant CPAN CSD2007-00042. T.D. also acknowledges the support of the MICINN under grant FPA2009-08958, the Community of Madrid under grant HEPHACOS S2009/ESP-1473, and the European Union under the Marie Curie-ITN program PITN-GA-2009-237920. Y.Y.K. would like to thank LAPTh in Annecy, France, for the warm hospitality during his visits there. His work is partially supported by the Research Professorship (A) of Ewha Womans University during 2010-2011, and by the Basic Science Research Program through the National Research Foundation of Korea (NRF) funded by the Korean Ministry of Education, Science and Technology (Grant No: 2009-0090848). W.L. would like to thank the staff in LAPTh for their hospitality during one year and the LAPTh partial financial support. He would like to thank Xuelei Chen, Youjun Lu, and Xiaoxia Zhang for helpful discussions. He is grateful to the China Scholarship Council for its financial support (No. 2009491052). P.S. expresses his gratitude to the Institut universitaire de France (IUF) for academically and financially supporting his research.

\section{References}

Adriani, O., Barbarino, G. C., Bazilevskaya, G. A., et al. 2011, Science, 332, 69 Ahn, H. S., Allison, P., Bagliesi, M. G., et al. 2010, ApJ, 714, L89
Ave, M., Boyle, P. J., Höppner, C., Marshall, J., \& Müller, D. 2009, ApJ, 697, 106

Bernard, G., Delahaye, T., Salati, P., \& Taillet, R. 2012, A\&A, 544, A92

Biermann, P. L., Becker, J. K., Dreyer, J., et al. 2010, ApJ, 725, 15

Blasi, P., \& Amato, E. 2011, J. Cosmol. Astropart. Phys., 2012, 28

Blasi, P., Amato, E., \& Serpico, P. D. 2012, Phys. Rev. Lett., 109, 061101

Bringmann, T., Donato, F., \& Lineros, R. A. 2012, J. Cosmol. Astropart. Phys., 2012, 049

Diehl, R., Halloin, H., Kretschmer, K., et al. 2006, Nature, 439, 45

Donato, F., Fornengo, N., Maurin, D., \& Salati, P. 2004, Phys. Rev., 69, 3501

Erlykin, A. D., \& Wolfendale, A. W. 2011, Astropart. Phys., 35, 14

Green, D. A. 2009, Bull. Astron. Soc. India, 37, 45

Hörandel, J. R., Kalmykov, N. N., \& Timokhin, A. V. 2007, Astropart. Phys., 27, 119

Malkov, M., Diamond, P., \& Sagdeev, R. 2012, Phys. Rev. Lett., 108, 1104

Manchester, R. N., Hobbs, G. B., Teoh, A., \& Hobbs, M. 2005, Astron. J., 129, 1993

Maurin, D., Donato, F., Taillet, R., \& Salati, P. 2001, ApJ, 555, 19

Maurin, D., Putze, A., \& Derome, L. 2010, A\&A, 516, A67

Nakamura, K., Particle Data Group, et al. 2010, J. Phys., 37, 5021

Norbury, J. W., \& Townsend, L. W. 2007, Nucl. Instrum. Meth., B254, 187

Ohira, Y., \& Ioka, K. 2011, ApJ, 729, L13

Putze, A., Derome, L., \& Maurin, D. 2010, A\&A, 516, 20

Stanev, T., Biermann, P. L., \& Gaisser, T. K. 1993, A\&A, 274, 902

Strong, A. W., Porter, T. A., Digel, S. W., et al. 2010, ApJ, 722, 7

Taillet, R., Salati, P., Maurin, D., Vangioni-Flam, E., \& Cassé, M. 2004, ApJ, 609,173

Thoudam, S., \& Hörandel, J. R. 2012, MNRAS, 421, 1209

Tomassetti, N. 2012, ApJ, 752, L13

Vladimirov, A. E., Jóhannesson, G., Moskalenko, I. V., \& Porter, T. A. 2012, ApJ, 752, 68

Yoon, Y. S., Ahn, H. S., Allison, P. S., et al. 2011, ApJ, 728, 20

Yuan, Q., Zhang, B., \& Bi, X.-J. 2011, in Contribution to the 32nd ICRC, Beijing, \#1355, 4

Zatsepin, V. I., \& Sokolskaya, N. V. 2006, A\&A, 458, 5 